\title{
Non-Visit-Based Cancer Screening Using a Novel Population Management System
}

\author{
Steven J. Atlas, MD, MPH, Adrian H. Zai, MD, PhD, MPH, Jeffrey M. Ashburner, MPH, \\ Yuchiao Chang, PhD, Sanja Percac-Lima, MD, PhD, Douglas E. Levy, PhD, \\ Henry C. Chueh, MD, MS, and Richard W. Grant, MD, MPH
}

Background: Advances in information technology (IT) now permit population-based preventive screening, but the best methods remain uncertain. We evaluated whether involving primary care providers (PCPs) in a visit-independent population management IT application led to more effective cancer screening.

Methods: We conducted a cluster-randomized trial involving 18 primary care practice sites and 169 PCPs from June 15, 2011, to June 14, 2012. Participants included adults eligible for breast, cervical, and/or colorectal cancer screening. In practices randomized to the intervention group, PCPs reviewed real-time rosters of their patients overdue for screening and provided individualized contact (via a letter, practice delegate, or patient navigator) or deferred screening (temporarily or permanently). In practices randomized to the comparison group, overdue patients were automatically sent reminder letters and transferred to practice delegate lists for follow-up. Intervention patients without PCP action within 8 weeks defaulted to the automated control version. The primary outcome was adjusted average cancer screening completion rates over 1-year follow-up, accounting for clustering by physician or practice.

Results: Baseline cancer screening rates $(\mathbf{8 0 . 8 \%}$ vs $80.3 \%)$ were similar among patients in the intervention $(n=51,071)$ and comparison group $(n=52,799)$. Most intervention providers used the IT application (88 of 101, 87\%) and users reviewed 7984 patients overdue for at least 1 cancer screening (73\% sent reminder letter, $6 \%$ referred directly to a practice delegate or patient navigator, and $21 \%$ deferred screening). In addition, 6128 letters were automatically sent to patients in the intervention group (total of 12,002 letters vs 16,378 letters in comparison practices; $P<.001$ ). Adjusted average cancer screening rates did not differ among intervention and comparison practices for all cancers combined $(81.6 \%$ vs $81.4 \% ; P=.84)$ nor breast $(82.7 \%$ vs $82.7 \% ; P=.96)$, cervical $(84.1 \%$ vs $84.7 \% ; P=$ $.60)$, or colorectal cancer $(77.8 \%$ vs $76.2 \% ; P=.33)$.

Conclusions: Involving PCPs in a visit-independent population management IT application resulted in similar cancer screening rates compared with an automated reminder system, but fewer patients were sent reminder letters. This suggests that PCPs were able to identify and exclude from contact patients who would have received automated reminder letters but not undergone screening.( $\mathrm{J}$ Am Board Fam Med 2014;27:474-485.)

Keywords: Cancer Screening, Prevention, Primary Health Care

Screening asymptomatic adults for breast, cervical, and colorectal cancer is widely recommended, ${ }^{1-4}$ but not all individuals who are eligible undergo

This article was externally peer reviewed.

Submitted 12 December 2013; revised 27 March 2014; accepted 31 March 2014.

From the General Medicine Division, Medical Services (SJA, JMA, YC, SP-L), the Laboratory of Computer Science (AHZ, HCC), and the Mongan Institute for Health Policy (DEL), Massachusetts General Hospital, Harvard Medical School, Boston, MA; and the Division of Research, Kaiser Permanente Northern California, Oakland (RWG). testing. ${ }^{5,6}$ Electronic health record (EHR) reminders associated with office visits have been shown to modestly increase preventive cancer screening Agency for Healthcare Research and Quality (AHRQ R18HS018161), the Controlled Risk Insurance Company/Risk Management Foundation, and institutional funding through the Massachusetts General Hospital Primary Care Operations Improvement Program and the Massachusetts General Physicians Organization.

Conflict of interest: Massachusetts General Hospital entered into a royalty arrangement on June 27, 2013, to commercialize the population management system with SRG 
rates. $^{7-9}$ However, time constraints for clinicians during office visits and patients who do not pursue follow-up limit the effectiveness of office-based systems and have led to increased efforts to screen patients outside of office visits. ${ }^{10,11}$

Non-visit-based reminder systems for cancer screening, including those using health information technology (IT), have been shown to increase screening rates but generally focus on only a single condition. ${ }^{12-17}$ We showed improved screening rates using a population management system in which primary care providers (PCPs) reviewed a list of their patients overdue for breast cancer screening and, if needed, took action to initiate a cascade of downstream events. ${ }^{18,19}$ This model of care emphasizes management outside of office visits and provides a framework to facilitate the efficient allocation of limited clinical resources. ${ }^{20,21}$

Advances in IT and new payment models encourage greater population-level oversight for comprehensive cancer screening outside of the office visit, but the best ways to do this remain uncertain. $^{22}$ Therefore, we designed a visit-independent population management IT application to support preventive screening for breast, cervical, and colorectal cancer within a primary care practice network. We implemented this application as part of a practice-randomized trial that compared involving PCPs in the screening process to customize outreach with a fully automated outreach process. Although higher cancer screening rates are associated with having a $\mathrm{PCP}^{23,24}$ and the PCP recommending screening, ${ }^{25-28}$ the role of the PCP in non-visit-based outreach efforts is unclear. We hypothesized that having PCPs determine whether contact was needed-and, if needed, how best to provide it-would lead to an increase in cancer screening rates. In this article we present cancer screening test completion rates over 1 year of follow-up comparing these 2 screening strategies.

Technology, a for-profit company. AHZ, HCC, and SJA are beneficiaries of this royalty arrangement but have not received any payments to date. AHZ, HCC, and SJA are in negotiations with SRG Technology, a for-profit company, regarding engagement as consultants in disseminating the population management system through this company. No consulting agreement has been finalized, and no payments have been provided.

Corresponding author: Steven J. Atlas, MD, General Medicine Division, Massachusetts General Hospital, 50 Staniford Street, Boston, MA 02114 (E-mail: satlas@partners. org).

\section{Methods}

\section{Study Design and Randomization}

We conducted a cluster randomized clinical trial involving 18 primary care practice sites. Since the intervention was implemented at the practice level, we randomly assigned practices to intervention $(\mathrm{n}=9)$ or comparison $(\mathrm{n}=9)$ groups. To minimize imbalance between groups, practices were first stratified by practice type (community health center or non-health center [includes both hospitalbased and neighborhood practices]). Within each type, practice pairs were matched by prior year baseline screening rates for all 3 cancers, cancer screening rates in unaffiliated outside facilities, the total number of eligible patients, the proportion of each sex in the patient panel, and the proportion of patients linked with a specific physician. ${ }^{29}$ Practices within each matched pair were then randomly allocated to intervention versus comparison arms. The Massachusetts General Hospital Institutional Review Board approved the study.

\section{Setting and Participants}

The study took place in the Massachusetts General Primary Care Practice-Based Research Network. All practices in the network use EHRs with information about preventive cancer screening tests available during clinical encounters. Eligible patients had at least one visit to a study practice within the prior 3 years or during the 1-year study period and were connected with a specific network physician (either a family physician, general internist, or medicine/pediatrics physician) or practice. ${ }^{30}$ Patients eligible for breast, cervical, and/or colorectal cancer screening included women 42 to 74 years of age who had not undergone bilateral mastectomy (women 42 to 49 years of age were advised to consider screening based on current recommendations), ${ }^{29}$ women 21 to 64 years of age who had not undergone total hysterectomy, and men or women 52 to 75 years of age who had not undergone total colectomy, respectively (Figure 1). Patients were considered overdue for screening if they were eligible for breast cancer screening but had no record of having a mammogram in the past 2 years, were eligible for cervical cancer screening but had no record of having a Papanicolaou test in the past 3 years, and/or were eligible for colorectal cancer screening but had no record of having a colonoscopy in the past 10 years or sigmoidoscopy, 
Figure 1. CONSORT diagram depicting the flow of study practice clusters and patients through randomization, intervention, and outcome analysis.

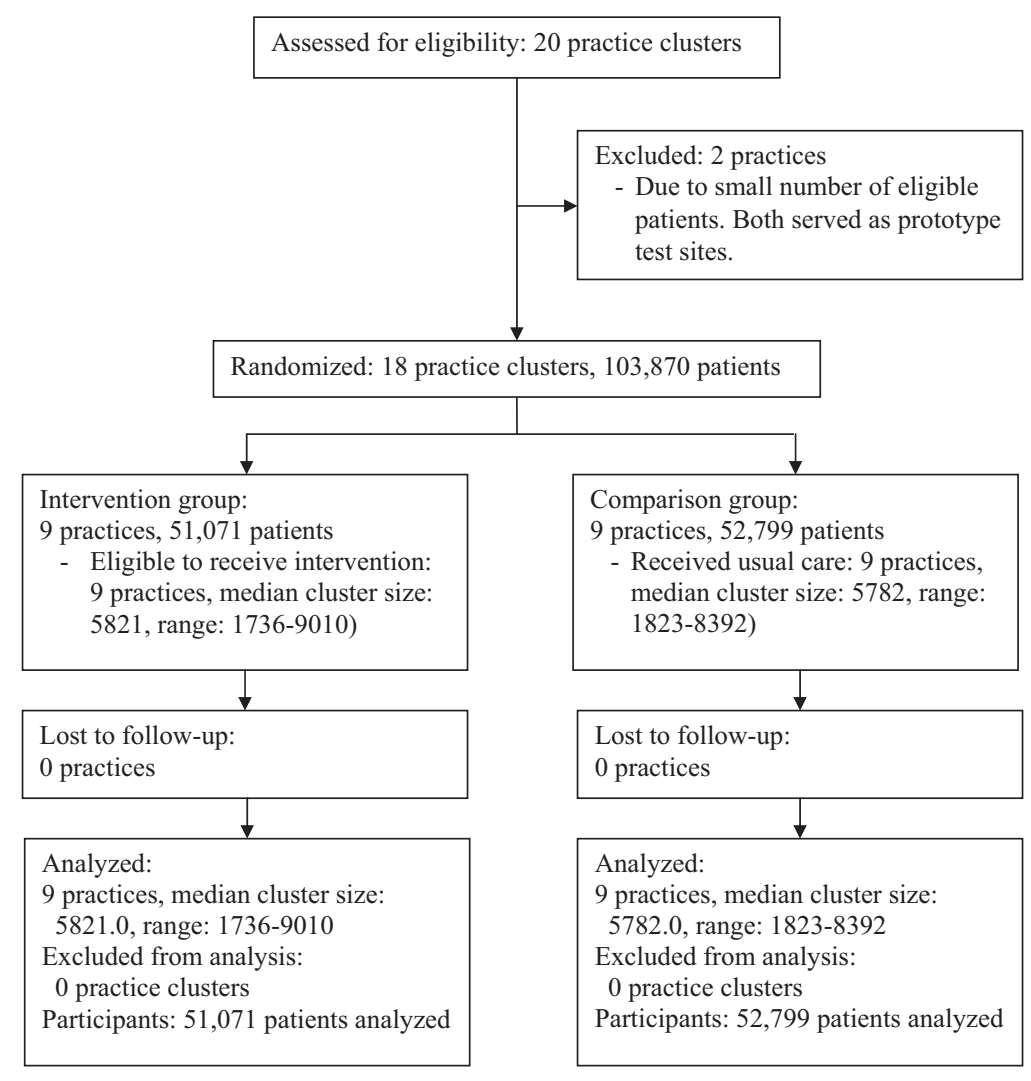

barium enema, or computed tomography colonography in the past 5 years. No practices used fecal immunochemical testing, but home fecal occult blood testing was an option for some patients who refused other methods of colorectal cancer screening. However, poor documentation of completed home fecal occult blood testing led to not using it as part of the outcome measure.

\section{Interventions}

The web-based IT application ${ }^{31}$ was implemented in all study practices for a 1-year period from June 15, 2011, until June 14, 2012. The IT application functions independently of the EHR by retrieving information from the EHR, scheduling/billing systems, and laboratory/test databases.

\section{Comparison Arm (Augmented Usual Care)}

Comparison group practices used an automated version of the IT application without provider review. The automated IT application performed 3 broad functions (Figure 2): (1) it identified all patients overdue for breast, cervical, and/or colorectal

cancer screening in real time and mailed them customized reminder letters (in English or Spanish based on the language preference in the patient registration system) with informational handouts; (2) it transferred them to a practice delegate (this role is defined in the "Practice Delegate Role" section) who could use the application to assist with scheduling tests; and (3) it tracked scheduled and completed testing so that only patients with unscheduled tests were displayed. In addition, patients remaining on practice delegate lists for at least 4 months and who were identified as being at increased risk for nonadherence to screening (via an algorithm using patient age, non-English speaking, number of overdue tests, and no-show visits) were automatically transferred from the practice delegate list to a central patient navigator list for further outreach.

\section{Intervention Arm (Augmented Usual Care with Provider Input)}

Intervention practices used the same IT application, except providers could customize individual 
Figure 2. Diagram depicting the workflow of intervention and comparison groups. Augmented usual care with primary care provider (PCP) input (intervention) is indicated by solid lines; augmented usual care without PCP input (comparison) is indicated by dotted lines.

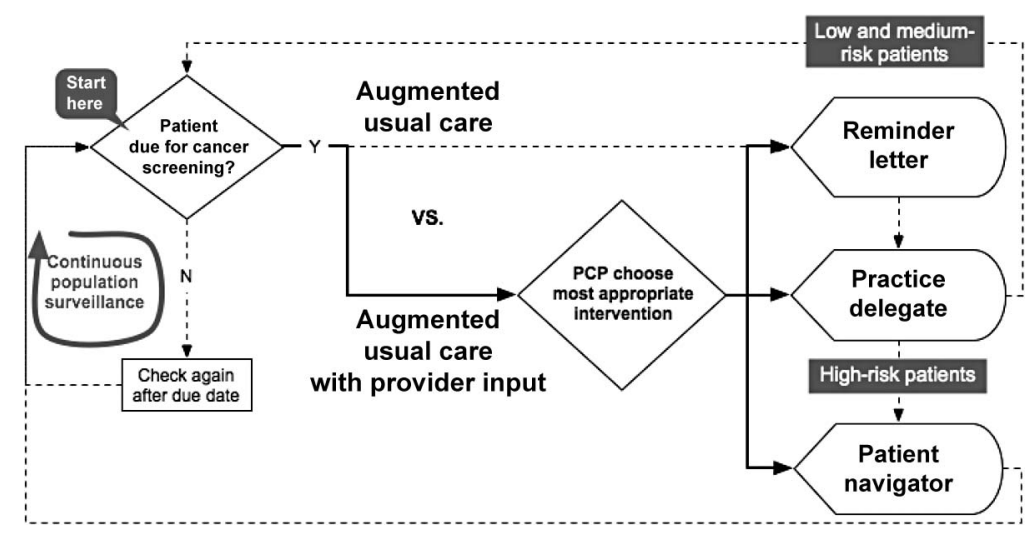

patient outreach. Physicians (for patients linked to a physician) and population managers (for patients not linked to a physician) used the IT application to review a list of their overdue patients (Figure 2). This roster was updated daily and was accessed via monthly E-mail reminders to users or any time via links within the EHR. The provider roster included (1) patient identifiers; (2) eligible cancer screening test(s) and screening status (to be listed when at least one test was overdue); (3) clinically relevant decision support retrieved from various electronic databases, including any documented information about cancer screenings completed outside the network, to help providers decide whether to initiate patient contact; and (4) an actionable component that allowed the provider, with "one click," to mail a reminder letter, directly transfer to a practice delegate for prioritized outreach, directly transfer to a patient navigator for intensive outreach, or defer screening either temporarily or permanently. If more information was required, the application provided a direct link to the EHR. Providers had 8 weeks to take action, and their list displayed the number of days remaining before a patient was transferred to the automated system, which operated in the same manner as in the comparison practices.

\section{Practice Delegate Role}

Practice delegates (either administrative staff or medical assistants) from both intervention and comparison practices facilitated follow-up among overdue patients. Patients appeared on practice delegate lists after being sent a reminder letter or after a provider from an intervention practice directly referred them. Letters included a practice phone number so patients could call the practice delegate directly and request assistance with scheduling. Practice delegates could also use the list to make outgoing calls to patients, and those in intervention practices were trained to give priority to patients referred directly by providers. Patients who scheduled or completed all overdue tests were automatically removed from practice delegate lists, but patients who missed scheduled screening appointments were added back. After 4 months, patients still overdue were transferred to a patient navigator list if a decision-support algorithm identified them as at increased risk for screening nonadherence. All other patients were removed from the active system before becoming eligible again after 8 months had elapsed and if overdue for screening.

\section{Patient Navigator Role}

The ability to navigate patients referred by intervention providers or from the risk algorithm for screening nonadherence for all sites began in October 2011 (it was delayed because of hiring issues) using a full-time trilingual (English, Spanish, Portuguese) patient navigator. Other existing navigator and interpreter resources were used to help patients who spoke any of 18 other languages. Patient navigators provided more intense outreach than practice delegates, including frequently attempting contact, exploring individual barriers to screening, educating patients, providing reminder calls, arranging transportation, assisting with visit preparation, and accompanying patients to visits. ${ }^{32-34} \mathrm{~Pa}$ - 
tients remained on the navigator roster until all overdue tests were completed or the patient navigator selected an appropriate reason for deferral or exclusion.

\section{Outcome Measures}

Patient characteristics and cancer screening data were obtained from an electronic central data repository at Partners Health Care. ${ }^{35}$ Dates of completion for mammograms, Papanicolaou tests, and colorectal cancer screening tests were obtained from electronic reports or billing data. Physician characteristics were obtained from the hospital registrar. The IT application collected information on usage by providers, practice delegates, and patient navigators.

The primary outcome was the overall cancer screening test completion rate over the 1-year follow-up period for each eligible patient, with all eligible cancers combined. For example, a patient who was eligible for a total of 3 screening tests at a given time could have a completion rate of $0 \%$ (none of the 3 tests completed), $33 \%, 67 \%$, or $100 \%$ (all 3 tests completed). Similarly, the completion rate could be $0 \%, 50 \%$, or $100 \%$ if patients were eligible for 2 screening tests at a given time. By assessing each patient's completion rate over the 1-year follow-up period, the average completion rate over time was estimated from the area under the curve. We also calculated the completion rate for each individual cancer as the percentage of time screening was up to date among eligible patients during follow-up. Secondary outcomes included comparisons of average cancer screening test completion in specified subgroups, including individuals newly overdue for screening during the 1-year study period and in practices with more practice delegate outreach activity. Other outcomes included measures of IT application usage by practice delegates (documented calls, referrals, exclusions) and intervention provider actions (reminder letters sent, deferrals, exclusions). Physician surveys were administered before and 1 year after implementation of the IT application to assess reported time spent on and satisfaction with cancer screening tasks.

\section{Statistical Analyses}

We compared patient and physician/practice characteristics between the intervention and comparison groups using 2-sample $t$ tests or $\chi^{2}$ tests, as appropriate. For primary and secondary outcomes, a mixed effects model was used to compare the average completion rate between patients from intervention practices and patients from comparison practices for all cancer screening exams combined and for each individual screening examination while taking into account clustering by PCP or practice (PROC MIXED function, SAS version 9.2; SAS Institute, Cary, NC). The physician was considered as the unit of cluster for patients connected with a specific PCP, and the practice was considered the unit of cluster for patients who could be connected with a practice but not a specific PCP. To control for differences in patients and practice characteristics among intervention and comparison practices, age, ethnicity, insurance status, primary language, time since last practice visit, and sex (for all cancer screenings combined and for colorectal cancer screening) were included in the models as covariates. We compared the primary outcomes in intervention and comparison practices within relevant subgroups and calculated adjusted rate differences and $95 \%$ confidence intervals. Patient subgroups were defined by age, number of overdue tests in women, race/ethnicity, English language proficiency, and insurance status. Physician/system subgroups included patient-provider linkage status, practice site (health center or not), and engagement of the IT application by providers and practice delegates. Physician survey responses before and after the survey were compared using the McNemar's $\chi^{2}$ test.

The study was powered so that a sample size of 45,000 patients in each group (equivalent to an effective sample size of 3,300 patients per group after accounting for clustering) would have $90 \%$ power to detect a $1.3 \%$ difference in mean completion rate over the follow-up period, assuming a common standard deviation of $16 \%$.

\section{Results}

\section{Practice, Physician, and Patient Characteristics}

Practice, physician, and patient characteristics are shown in Table 1. Three community health center sites were in each study arm. There were 92 physicians in the 9 intervention arm practices and 77 physicians in the 9 comparison arm practices. Intervention physicians were slightly more experienced, but differences were not statistically significant. A total of 103,870 patients were eli- 
Table 1. Practice, Physician, and Patient Characteristics among Intervention and Comparison Practices

\begin{tabular}{|c|c|c|c|}
\hline Characteristics & Intervention $(\mathrm{n}=51,071)$ & Comparison $(\mathrm{n}=52,799)$ & $P$ Value \\
\hline \multicolumn{4}{|l|}{ Practice/physician characteristics } \\
\hline Practice sites (n) & 9 & 9 & \\
\hline Community health center & 3 & 3 & \\
\hline Physicians per practice, $\mathrm{n}$ (median) & $92(9)$ & $77(9)$ & \\
\hline Mean age, years (SD) & $49.7(10.1)$ & $47.5(10.0)$ & .14 \\
\hline Sex & $50(54.4)$ & $45(58.4)$ & .59 \\
\hline Years since medical school graduation, mean (SD) & $21.8(10.4)$ & $19.6(10.1)$ & .15 \\
\hline Years in primary care network, mean (SD) & $15.8(10.9)$ & $13.1(10.4)$ & .09 \\
\hline \multicolumn{4}{|l|}{ Patient characteristics } \\
\hline Mean age, years (SD) & $51.5(14.3)$ & $48.5(14.8)$ & $<.001$ \\
\hline Female sex & $37,906(74.2)$ & $40,568(76.8)$ & $<.001$ \\
\hline Ethnicity & & & $<.001$ \\
\hline African-American & $2920(5.7)$ & $3319(6.3)$ & \\
\hline Asian & $2724(5.3)$ & $3473(6.6)$ & \\
\hline Hispanic & $3865(7.6)$ & $5964(11.3)$ & \\
\hline Other/unknown & $1256(2.5)$ & $1384(2.6)$ & \\
\hline Non-Hispanic white & $40,306(78.9)$ & $38,659(73.2)$ & \\
\hline Insurance status & & & $<.001$ \\
\hline Commercial & $35,665(69.8)$ & $37,895(71.8)$ & \\
\hline Medicaid & $4602(9.0)$ & $5486(10.4)$ & \\
\hline Medicare & $9058(17.7)$ & $7437(14.1)$ & \\
\hline No insurance, self-pay/free & $1746(3.4)$ & $1981(3.8)$ & \\
\hline Primary language spoken, English & $46,560(91.2)$ & $46,478(88.0)$ & $<.001$ \\
\hline Patient-physician connectedness status & & & $<.001$ \\
\hline Physician-connected & $42,449(83.1)$ & $42,132(79.8)$ & \\
\hline Practice-connected & $8622(16.9)$ & $10,667(20.2)$ & \\
\hline Time since last practice visit (months) & & & $<.001$ \\
\hline$<6$ & $31,439(61.6)$ & $30,658(58.1)$ & \\
\hline$>6-12$ & $10,206(20.0)$ & $10,473(19.8)$ & \\
\hline$>12$ & $6668(13.1)$ & $8663(16.4)$ & \\
\hline New patient & $2758(5.4)$ & $3005(5.7)$ & \\
\hline Community health center practice type & $7008(13.7)$ & $8935(16.9)$ & $<.001$ \\
\hline \multicolumn{4}{|l|}{ Baseline screening rates, $\mathrm{n} / \mathrm{N}(\%)$} \\
\hline Breast cancer & $18,389 / 22,425(82.0)$ & $16,556 / 20,439(81.0)$ & .01 \\
\hline Cervical cancer & $23,031 / 27,748(83.0)$ & $26,847 / 31,961(84.0)$ & .001 \\
\hline Colorectal cancer & $20,642 / 26,843(76.9)$ & $17,431 / 23,556(74.0)$ & $<.001$ \\
\hline
\end{tabular}

Data are $\mathrm{n}(\%)$ unless otherwise indicated. SD, standard deviation.

gible for at least 1 cancer screening during the 1-year study period (intervention group: 51,071; comparison group: 52,799). Patients in intervention practices were slightly older and slightly more likely to be male, non-Hispanic white, to speak English, to have a primary care visit within the past 6 months, to be connected to a specific $\mathrm{PCP}$, and to have Medicare insurance (all $P<$ .001). Intervention patients were slightly less likely to have commercial insurance and to be seen in a community health center $(P<.001)$.

\section{Use of the IT Application}

All intervention population managers (9 of 9 , $100 \%)$ and 79 of 92 intervention physicians (85.9\%) used the IT application during the study period. A total of 16,573 patients who seemed to be overdue for at least one cancer screening test were sent to intervention provider rosters over the study period. Providers reviewed and took action on 7984 (48.2\%) patients (5874 [73.6\%] selected to send a reminder letter, $401[5.0 \%]$ referred directly to a practice delegate, 47 [0.6\%] referred directly to a 
Table 2. Cancer Screening Rates Among Intervention and Comparison Group Patients Eligible for at Least 1 Cancer Screening Test During the Study Period

\begin{tabular}{|c|c|c|c|c|c|c|c|c|}
\hline \multirow[b]{4}{*}{ Cancer Screening } & \multicolumn{8}{|c|}{ Average Cancer Screening Test Completion Rates } \\
\hline & \multicolumn{5}{|c|}{ Unadjusted } & & & \\
\hline & \multicolumn{2}{|c|}{ Intervention } & \multicolumn{2}{|c|}{ Comparison } & \multirow[b]{2}{*}{$P$ Value } & \multicolumn{3}{|c|}{ Adjusted* } \\
\hline & No. & Rate (\%) & No. & Rate (\%) & & Intervention (\%) & Comparison (\%) & $P$ Value \\
\hline All eligible cancers & 51,071 & 81.6 & 52,799 & 81.4 & .90 & 81.6 & 81.4 & .84 \\
\hline Breast & 24,602 & 82.8 & 22,351 & 82.7 & .93 & 82.7 & 82.7 & .96 \\
\hline Cervical & 32,121 & 84.2 & 35,889 & 84.7 & .72 & 84.1 & 84.7 & .60 \\
\hline Colorectal & 30,353 & 77.9 & 26,756 & 76.2 & .33 & 77.8 & 76.2 & .33 \\
\hline
\end{tabular}

*Adjusted rates and $P$ values obtained from mixed effects models comparing intervention and control groups controlling for patient age, ethnicity, insurance status, primary language, time since last visit to practice, patient-physician linkage, and sex (for colorectal cancer and all screenings combined) while accounting for clustering by primary care physician or practice in a mixed effects model.

patient navigator, 1551 [19.4\%] deferred temporarily, and 111 [1.4\%] excluded permanently). Among intervention patients for whom no action was taken by their provider, $6128(37.0 \%)$ were sent automated reminder letters, $1764(10.6 \%)$ who were no longer overdue were removed by the system before a letter was mailed, and 697 (4.2\%) patients remained on provider rosters at the end of the study period. In comparison practices, 16,378 patient letters were mailed without provider review $(31.0 \%$ [16,378 of 52,799] of eligible patients) compared with a total of 12,002 letters in intervention practices $(23.5 \%$ [12,002 of 51,071$]$ of eligible patients; $P<.001)$. Overall, practice delegates documented actions (calls, deferrals, and exclusions) among $4.7 \%$ of patients on their rosters. In the intervention group, practice delegates documented actions in $6.6 \%$ of patients (range by practice, $0-28.0 \%$ ), whereas in the comparison group, practice delegates documented actions in $3.4 \%$ of patients (range by practice, $0.2-8.2 \%$ ).

\section{Primary Outcomes: Cancer Screening Rates at Study Completion}

Among patients eligible for cancer screening, unadjusted and adjusted average cancer screening completion rates were similar (Table 2). There was no difference in adjusted average cancer screening test completion rates between the intervention and comparison groups for all cancers combined (intervention: $81.6 \%$, comparison: $81.4 \% ; P=.84$ ), breast cancer screening (intervention: $82.7 \%$, comparison: $82.7 \% ; P=.96$ ), cervical cancer screening (intervention: $84.1 \%$, comparison: $84.7 \% ; P=$
.60), or colorectal cancer screening (intervention: $77.8 \%$, comparison: $76.2 \% ; P=.33$ ).

\section{Secondary and Subgroup Analyses}

Among patients who seemed to be overdue for at least one cancer screening test during the study period, unadjusted and adjusted average cancer screening completion rates were similar in the intervention and comparison groups (Table 3). Because documented use of the IT application by practice delegates was low, exploratory analyses examined outcomes in practices where use of practice delegates was higher, since their involvement was hypothesized to have more of an effect in intervention practices. Among practices in the top tertile of IT application use by practice delegates, intervention patients had higher adjusted average cancer screening test completion rates for all cancers combined $(P<.001)$, breast cancer screening $(P=.06)$, and cervical cancer screening $(P<.001)$ but not colorectal cancer screening $(P=$ .79) (Table 3). However, overdue patients of intervention providers in the top tertile of IT application use did not have higher screening rates than overdue patients from comparison practices unless those intervention PCPs also were associated with higher rates of IT application use by practice delegates (data not shown).

The overall adjusted rate of a patient completing all eligible cancer screening tests was similar in both the intervention and comparison groups (rate difference, $0.25 \%$; $95 \%$ confidence interval, $-2.18 \%$ to $2.67 \%)$. There was no patient, physician, or practice subgroups in which the intervention was more effective (Figure 3). Among subgroup comparisons 
Table 3. Cancer Screening Rates among Eligible Intervention and Comparison Group Patients Overdue for at Least 1 Cancer Screening Test During the Study Period

\begin{tabular}{|c|c|c|c|c|c|c|c|c|}
\hline & \multicolumn{8}{|c|}{ Average Cancer Screening Test Completion Rates } \\
\hline & \multicolumn{5}{|c|}{ Unadjusted } & \multirow{2}{*}{\multicolumn{3}{|c|}{ Adjusted* $^{*}$}} \\
\hline & \multicolumn{2}{|c|}{ Intervention } & \multicolumn{2}{|c|}{ Comparison } & \multirow[b]{2}{*}{$P$ Value } & & & \\
\hline & No. & Rate $(\%)$ & No. & Rate $(\%)$ & & Intervention (\%) & Comparison (\%) & $P$ Value \\
\hline All eligible cancers & 18,873 & 18.3 & 19,201 & 17.8 & .59 & $18.8 \%$ & $17.8 \%$ & .28 \\
\hline Breast & 6927 & 23.2 & 6486 & 24.0 & .52 & $23.7 \%$ & $24.0 \%$ & .80 \\
\hline Cervical & 8919 & 23.3 & 9640 & 21.1 & .23 & $23.4 \%$ & $21.1 \%$ & .14 \\
\hline Colorectal & 8135 & 8.7 & 7740 & 9.3 & .29 & $9.0 \%$ & $9.3 \%$ & .53 \\
\hline \multicolumn{9}{|c|}{$\begin{array}{l}\text { Practices in top tertile of TopCare } \\
\text { practice delegate use }\end{array}$} \\
\hline All eligible cancers & 6276 & 22.3 & 7678 & 16.9 & .003 & 20.8 & 16.9 & $<.001$ \\
\hline Breast & 2503 & 26.5 & 2811 & 23.1 & .06 & 26.4 & 23.1 & .06 \\
\hline Cervical & 3166 & 28.4 & 3599 & 18.7 & .002 & 28.2 & 18.7 & $<.001$ \\
\hline Colorectal & 2279 & 9.3 & 3262 & 9.4 & .92 & 9.7 & 9.4 & .79 \\
\hline
\end{tabular}

*Adjusted rates and $P$ values obtained from mixed effects models comparing intervention and control groups controlling for patient age, ethnicity, insurance status, primary language, time since last visit to practice, patient-physician linkage, and sex (for colorectal cancer and all screenings combined) while accounting for clustering by primary care physician or practice in a mixed effects model.

for individual cancers, only the adjusted rate of breast cancer completion was higher among intervention patients compared with comparison patients seen in a health center (rate difference, $10.39 \%$; 95\% confidence interval $5.27-14.28 \%$; data not shown).

\section{Physician Time and Satisfaction with Cancer Screening Tasks}

Surveys were completed before and after the study by $49 \%$ (42 of 85 ) of physicians in intervention practices and $47 \%$ (34 of 72 ) of physicians in comparison practices. There were no significant differences before and after implementation in responses from PCPs in comparison practices. Among PCPs in the intervention group, the proportion who reported spending $<10$ minutes per clinical session devoted to cancer screening tasks increased for breast (before: $49 \%$, after: $58 \% ; P=.48$ ), cervical (before: $44 \%$, after: $65 \% ; P=.01$ ), and colorectal cancer screening (before: $26 \%$, after: $47 \% ; P=.05$ ). More intervention physicians believe the process for managing patients who are overdue for cancer screening improved over the past year (before: $21 \%$, after: $79 \% ; P<.001$ ). Among intervention PCPs who reviewed their roster and completed a survey after implementation (41 of $79,52 \%), 68 \%$ found the IT application to be easy to use, $63 \%$ indicated it made their time managing cancer screening more effective, and $88 \%$ were satisfied with the IT application.

\section{Discussion}

We compared cancer screening rates among patients eligible for breast, cervical, and/or colorectal cancer as part of a visit-independent, population management IT application within a primary care network. Over a 1-year study period, involving PCPs in the screening process did not increase testing rates compared with an automated reminder IT application that did not involve PCPs. However, similar screening rates were achieved with significantly fewer reminder letters in intervention practices where PCPs reviewed their overdue patients, and intervention PCPs reported spending less time during clinic hours on cancer screening tasks.

Studies have shown that involvement of a $\mathrm{PCP}^{23-28}$ and non-visit-based reminder systems ${ }^{10,12-17,36,37}$ can increase rates of preventive cancer screening. The current study demonstrates that an automated reminder IT application without physician input led to similar screening rates for patients who seemed to be overdue for up to 3 widely recommended cancers: breast, cervical, and colorectal. ${ }^{2,38,39} \mathrm{We}$ previously demonstrated that a similar population management system involving PCPs resulted in higher rates of breast cancer screening over a 3-year follow-up period compared with usual care that involved visit-based reminders in the EHR. ${ }^{18}$ The 1 -year results of 
Figure 3. Adjusted rate differences and $95 \%$ confidence intervals for all cancer screenings combined in intervention and comparison groups in patient and practice subgroups. Rate differences compare patients in the intervention and comparison groups, controlling for age, ethnicity, insurance status, primary language, time since last visit to practice, patient-physician linkage, and sex while accounting for clustering by primary care physician or practice in a mixed effects model. For each subgroup analysis, the analogous variable was removed from the model if necessary. CRC, colorectal cancer.

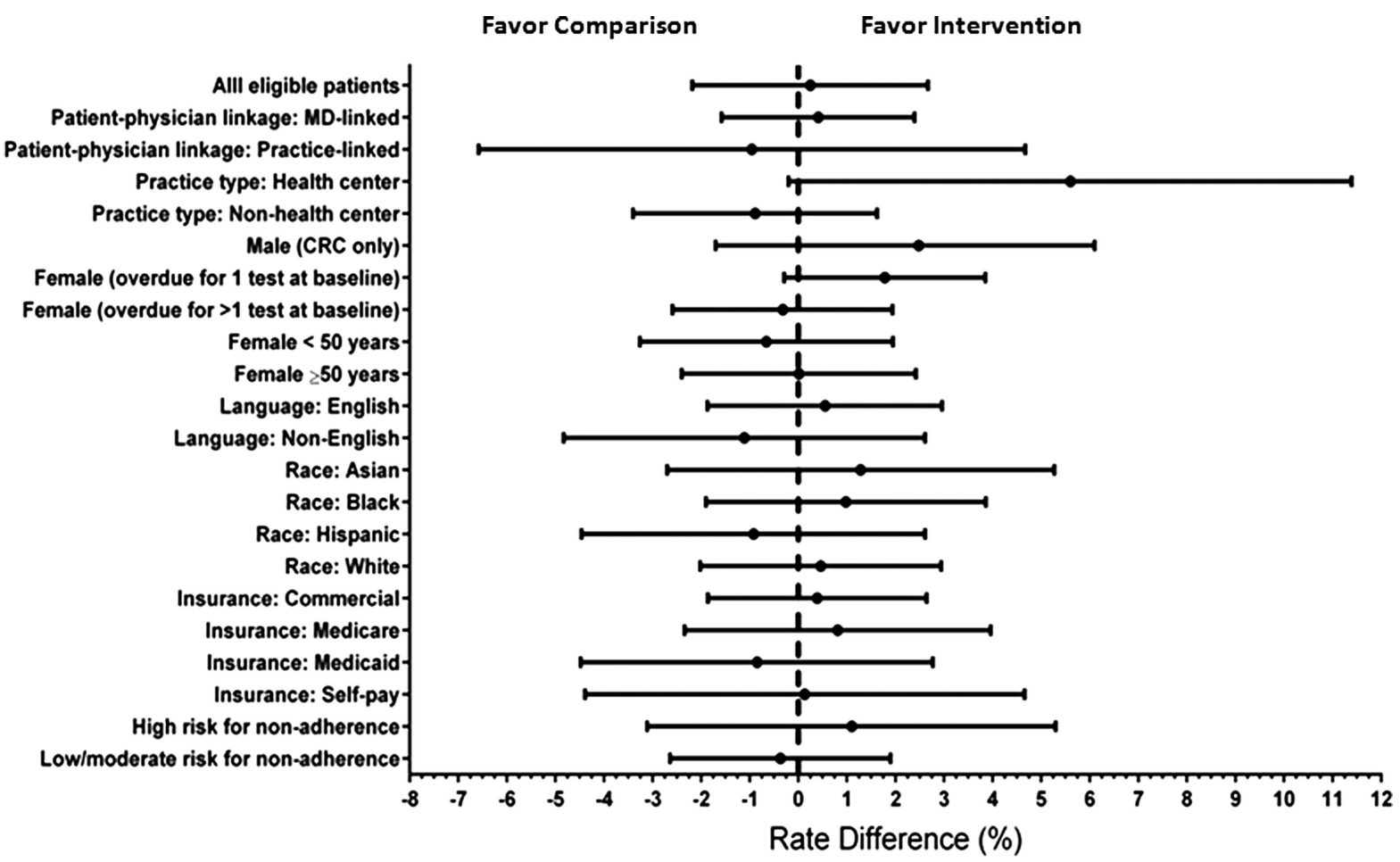

the intervention and comparison groups in the current study $(34.4 \%$ and $35.7 \%$ of patients who were overdue at baseline and followed for a 1 -year period were screened, respectively) are similar to those seen in the intervention group (31.4\%) of our prior breast cancer screening study, all of which are better than the rates seen in the control group from the prior study, which received usual care $(23.3 \%) .{ }^{19}$

Although physician involvement did not increase screening rates, fewer reminder letters were needed in intervention practices where PCPs reviewed their overdue list, implying that PCPs could accurately exclude patients who did not need or would not undergo screening. Moreover, in practices where PCPs were more likely to review overdue lists and practice delegates were more likely to document patient contacts, higher rates of screening occurred, especially for breast and cervical cancer. PCPs in intervention practices reported spending less time devoted to cancer screening tasks during clinical sessions and felt the process for managing patients who were overdue for cancer screening was improved. Future studies should examine the cost-effectiveness of involving PCPs in preventive cancer screening using population management systems.

Several important limitations are worth noting. First, our network had high baseline rates of preventive cancer screening, so it is possible that the small improvements in screening noted here may be larger in systems that start with lower rates of test completion. Second, although providers in intervention sites used the IT application to review overdue patients, practice delegates in both the intervention and comparison practices conducted limited patient outreach. This lack of practice delegate outreach may have undermined the hypothesis that involving PCPs would improve the effectiveness of care. Specifically, if PCPs identified individuals for targeted outreach as well as individuals who do not need screening (deferrals), then those provider's practice delegates would have "more accurate" lists 
and would be more likely to identify patients truly needing screening during their time commitment. Redesigning workflow to better involve practice delegates may increase the effect of PCP involvement. ${ }^{21,40}$ Third, it is possible that some patients who were not overdue for screening were sent reminders; however, we received no reports of such overtesting. Fourth, although similar percentages of intervention and comparison group PCPs completed surveys, low response rates could have led to biased results if reasons for not responding differed among groups. Finally, use of patient navigators by intervention PCPs was limited because the program was not in place at the start of the study. Future studies should examine how population management systems can efficiently use patient navigators for those patients who need more assistance to complete cancer screening. ${ }^{40}$

Our study did not evaluate whether non-visit based population management systems could be used to remove tasks from busy clinical encounters currently embedded in EHR reminders. ${ }^{41}$ As practices move to the patient-centered medical home model of team-based care, more of these routine activities likely will be performed outside of the office visit by nonclinical staff, thereby freeing up the time of clinical staff for other activities that may be harder to perform by others or outside of the office visit. ${ }^{42,43}$

To deliver better care at lower cost, current health care delivery models need to undergo a fundamental restructuring. ${ }^{44-46}$ Health IT, such as our population management IT application, offers the possibility of such transformational change. We demonstrated that an automated, non-visit-based IT application for comprehensive preventive cancer screening-one that identifies eligible individuals who are overdue for tests, contacts them, and then tracks them for test scheduling and completion-results in similar screening rates compared with a system that involves PCPs in identifying patients for outreach. However, such automated systems result in significantly more patient outreach, which may become burdensome to patients as these systems grow in scope and include a broad range of prevention and disease management registries and interventions. These results may help networks considering such non-visit-based reminder systems by identifying the tradeoffs between automated IT applications needlessly "hassling" pa- tients and the burden associated with providers prescreening their lists. Future research should seek to identify where provider input is critical, even when processes are highly automated.

\section{References}

1. U.S. Preventive Services Task Force. Screening for breast cancer: U.S. Preventive Services Task Force recommendations statement. Ann Intern Med 2009; 151:716-26.

2. Moyer VA; U.S. Preventive Services Task Force. Screening for cervical cancer: U.S. Preventive Services Task Force recommendation statement. Ann Intern Med 2012;156:880-91, W312.

3. Smith RA, Brooks D, Cokkinides V, et al. Cancer screening in the United States, 2013: a review of current American Cancer Society guidelines, current issues in cancer screening, and new guidance on cervical cancer screening and lung cancer screening. CA Cancer J Clin 2013;63:88-105.

4. Whitlock EP, Lin JS, Liles E, et al. Screening for colorectal cancer: a targeted, updated systematic review for the U.S. Preventive Services Task Force. Ann Intern Med 2008;149:638-58.

5. Centers for Disease Control and Prevention (CDC). Cancer screening-United States, 2010. MMWR Morb Mortal Wkly Rep 2012;61:41-5.

6. Carney PA, O'Malley J, Buckley DI, et al. Influence of health insurance coverage on breast, cervical, and colorectal cancer screening in rural primary care settings. Cancer 2012;118:6217-25.

7. Dexheimer JW, Talbot TR, Sanders DL, et al. Prompting clinicians about preventive care measures: a systematic review of randomized controlled trials. J Am Med Inform Assoc 2008;15:311-20.

8. Shea S, DuMouchel W, Bahamonde L. A metaanalysis of 16 randomized controlled trials to evaluate computer-based clinical reminder systems for preventive care in the ambulatory setting. J Am Med Inform Assoc 1996;3:399-409.

9. Shojania KG, Jennings A, Mayhew A, et al. Effect of point-of-care computer reminders on physician behaviour: a systematic review. CMAJ 2010;182:E216-25.

10. Steele RJ, Kostourou I, McClements P, et al. Effect of repeated invitations on uptake of colorectal cancer screening using faecal occult blood testing: analysis of prevalence and incidence screening. BMJ 2010; 341:c5531.

11. Yarnall KS, Pollak KI, Ostbye T, et al. Primary care: is there enough time for prevention? Am J Public Health 2003;93:635-41.

12. Chaudhry R, Scheitel SM, McMurtry EK, et al. Web-based proactive system to improve breast cancer screening: a randomized controlled trial. Arch Intern Med 2007;167:606-11.

13. Everett T, Bryant A, Griffin MF, et al. Interventions targeted at women to encourage the uptake of cer- 
vical screening. Cochrane Database Syst Rev 2011; (5):CD002834.

14. Green BB, Wang CY, Anderson ML, et al. An automated intervention with stepped increases in support to increase uptake of colorectal cancer screening: a randomized trial. Ann Intern Med 2013;158(5 Pt 1):301-11.

15. Sabatino SA, Lawrence B, Elder R, et al. Effectiveness of interventions to increase screening for breast, cervical, and colorectal cancers: nine updated systematic reviews for the guide to community preventive services. Am J Prev Med 2012;43:97-118.

16. Sequist TD, Zaslavsky AM, Marshall R, et al. Patient and physician reminders to promote colorectal cancer screening: a randomized controlled trial. Arch Intern Med 2009;169:364-71.

17. Yabroff KR, Zapka J, Klabunde CN, et al. Systems strategies to support cancer screening in U.S. primary care practice. Cancer Epidemiol Biomarkers Prev 2011;20:2471-9.

18. Atlas SJ, Ashburner JM, Chang Y, et al. Populationbased breast cancer screening in a primary care network. Am J Manag Care 2012;18:821-9.

19. Atlas SJ, Grant RW, Lester WT, et al. A clusterrandomized trial of a primary care informatics-based system for breast cancer screening. J Gen Intern Med 2011;26:154-61.

20. Lester WT, Zai AH, Grant RW, et al. Designing healthcare information technology to catalyse change in clinical care. Inform Prim Care 2008;16:9-19.

21. Zai AH, Grant RW, Estey G, et al. Lessons from implementing a combined workflow-informatics system for diabetes management. J Am Med Inform Assoc 2008; 15:524-33.

22. Lester WT, Ashburner JM, Grant RW, et al. Mammography FastTrack: an intervention to facilitate reminders for breast cancer screening across a heterogeneous multi-clinic primary care network. J Am Med Inform Assoc 2009;16:187-95.

23. Cardarelli R, Kurian AK, Pandya V. Having a personal healthcare provider and receipt of adequate cervical and breast cancer screening. J Am Board Fam Med 2010;23:75-81.

24. Cardarelli R, Thomas JE. Having a personal health care provider and receipt of colorectal cancer testing. Ann Fam Med 2009;7:5-10.

25. Fox SA, Murata PJ, Stein JA. The impact of physician compliance on screening mammography for older women. Arch Intern Med 1991;151:50-6.

26. Grady KE, Lemkau JP, McVay JM, et al. The importance of physician encouragement in breast cancer screening of older women. Prev Med 1992;21:766-80.

27. Metsch LR, McCoy CB, McCoy HV, et al. The role of the physician as an information source on mammography. Cancer Pract 1998;6:229-36.

28. Zarychanski R, Chen Y, Bernstein CN, et al. Frequency of colorectal cancer screening and the impact of family physicians on screening behaviour. CMAJ 2007;177:593-7.

29. Atlas SJ, Chang Y, Lasko TA, et al. Is this "my" patient? Development and validation of a predictive model to link patients to primary care providers. J Gen Intern Med 2006;21:973-8.

30. Atlas SJ, Grant RW, Ferris TG, et al. Patient-physician connectedness and quality of primary care. Ann Intern Med 2009;150:325-35.

31. SRG Technology. 2013. TopCare patient population management software. Fort Lauderdale, FL: SRG Tech, Inc. Available from: http://www.srgtech. com/topcare-population-health-management/. Accessed June 3, 2014.

32. Freeman HP. The origin, evolution, and principles of patient navigation. Cancer Epidemiol Biomarkers Prev 2012;21:1614-7.

33. Paskett ED, Harrop JP, Wells KJ. Patient navigation: an update on the state of the science. CA Cancer J Clin 2011;61:237-49.

34. Percac-Lima S, Grant RW, Green AR, et al. A culturally tailored navigator program for colorectal cancer screening in a community health center: a randomized, controlled trial. J Gen Intern Med 2009; 24:211-7.

35. Murphy SN, Chueh HC. A security architecture for query tools used to access large biomedical databases. Proc AMIA Symp 2002:552-6.

36. Muller D, Logan J, Dorr D, Mosen D. The effectiveness of a secure email reminder system for colorectal cancer screening. AMIA Annu Symp Proc 2009;2009:457-61.

37. Wagner TH. The effectiveness of mailed patient reminders on mammography screening: a meta-analysis. Am J Prev Med 1998;14:64-70.

38. Nelson HD, Tyne K, Naik A, et al. Screening for breast cancer: an update for the U.S. Preventive Services Task Force. Ann Intern Med 2009;151:72737, W237-42.

39. Qaseem A, Denberg TD, Hopkins RH Jr, et al. Screening for colorectal cancer: a guidance statement from the American College of Physicians. Ann Intern Med 2012;156:378-86.

40. Zai AH, Kim S, Kamis A, et al. Applying operations research to optimize a novel population management system for cancer screening. J Am Med Inform Assoc 2014;21(e1):e129-35.

41. Ayanian JZ, Sequist TD, Zaslavsky AM, et al. Physician reminders to promote surveillance colonoscopy for colorectal adenomas: a randomized controlled trial. J Gen Intern Med 2008;23:762-7.

42. Baker AN, Parsons M, Donnelly SM, et al. Improving colon cancer screening rates in primary care: a pilot study emphasising the role of the medical assistant. Qual Saf Health Care 2009;18:355-9.

43. Bates DW, Bitton A. The future of health information technology in the patient-centered medical home. Health Aff (Millwood) 2010;29:614-21. 
44. Lukas CV, Holmes SK, Cohen AB, et al. Transformational change in health care systems: an organizational model. Health Care Manage Rev 2007;32: 309-20.

45. Sepulveda MJ, Darling H. Large employers that have lived through transformation say payment re- form alone won't cut costs and reengineer care. Health Aff (Millwood) 2012;31:2037-42.

46. Wang MC, Hyun JK, Harrison M, et al. Redesigning health systems for quality: Lessons from emerging practices. Jt Comm J Qual Patient Saf 2006;32: $599-611$. 